\title{
Simplified Differential Equations Approach for Master Integrals
}

\author{
Costas G. Papadopoulos ${ }^{\star a}$, Damiano Tommasini ${ }^{a}$ and Christopher Wever ${ }^{a, b \dagger}$ \\ 'Institute of Nuclear and Particle Physics, NCSR 'Demokritos', Agia Paraskevi, 15310, Greece \\ ${ }^{b}$ Institute for Theoretical Particle Physics (TTP), Karlsruhe Institute of Technology, \\ Engesserstraße 7, D-76128 Karlsruhe, Germany \& Institute for Nuclear Physics (IKP), \\ Karlsruhe Institute of Technology, Hermann-von-Helmholtz-Platz 1, D-76344 \\ Eggenstein-Leopoldshafen, Germany \\ E-mail: costas.papadopoulos@cern.ch
}

In this talk we present the Simplified Differential Equations (SDE) Approach for Master Integrals (MI). Combined with the integrand reduction method for two-loop amplitudes it can pave the road for a fully automated NNLO calculation framework. Most recent achievements of the method, including double-box and pentabox MI calculations, are highlighted.

12th International Symposium on Radiative Corrections (Radcor 2015) and LoopFest XIV (Radiative Corrections for the LHC and Future Colliders)

15-19 June, 2015

UCLA Department of Physics Astronomy Los Angeles, USA

\footnotetext{
* Speaker.

${ }^{\dagger}$ This research was supported by the Research Funding Program ARISTEIA, HOCTools (co-financed by the European Union (European Social Fund ESF) and Greek national funds through the Operational Program "Education and Lifelong Learning" of the National Strategic Reference Framework (NSRF)).
} 


\section{Introduction}

With LHC delivering collisions at the highest energy achieved so far, $13 \mathrm{TeV}$, experiments are providing measurements of physical observables with an unprecedented precision. In order to keep up with the increasing experimental accuracy as more data is collected, more precise theoretical predictions and higher loop calculations are required [1].

With the better understanding of the reduction of one-loop amplitudes to a set of Master Integrals (MI) based on unitarity methods [2,3] and at the integrand level via the OPP method [4, 5], one-loop calculations have been fully automated in many numerical tools [6, 7]. In the recent years, a lot of progress has been made towards the extension of these reduction methods to the two-loop order at the integral $[8,9]$ as well as the integrand $[10,11,12,13]$ level. The master equation at the integrand level can be given schematically as follows [13]

$$
\frac{N\left(l_{1}, l_{2} ;\left\{p_{i}\right\}\right)}{D_{1} D_{2} \ldots D_{n}}=\sum_{m=1}^{\min (n, 8)} \sum_{S_{m ; n}} \frac{\Delta_{i_{1} i_{2} \ldots i_{m}}\left(l_{1}, l_{2} ;\left\{p_{i}\right\}\right)}{D_{i_{1}} D_{i_{2}} \ldots D_{i_{m}}} .
$$

where an arbitrary contribution to the two-loop amplitude (left), can be reduced to a sum of terms (right) of all partitions $S_{m ; n}$, with up to eight denominators; $l_{1}, l_{2}$ are the loop momenta, $D_{i}$ are the inverse scalar Feynman propagators, $N\left(l_{1}, l_{2} ;\left\{p_{i}\right\}\right)$ is a general numerator polynomial and $\Delta_{i_{1} i_{2} \ldots i_{m}}\left(l_{1}, l_{2} ;\left\{p_{i}\right\}\right)$ are the residues of multivariate polynomial division. In addition $R_{2}$ terms [5] have to be studied at two loops in order to achieve a complete framework. Moreover, two remarks are in order here. The first is that the basis of two-loop integrals does not include only scalar integrals. It includes integrals that also have irreducible scalar products (ISP) as numerators (to some power) that cannot be rewritten as existing denominators of the integral. In the one-loop case these ISP are always spurious and integrate to zero, but for higher loops this does no longer hold. The second remark is that if one is interested in constructing an integral basis, the set of integrals that ends up with is not necessarily a minimal one: the integrals are not by default Master Integrals. At two or more loops one can find them by using integration-by-parts (IBP) identities $[14,15,16,17,18]$.

Contrary to the MI at one-loop, which have been known for a long time already [19], a complete library of MI at two-loops is still missing. At the moment this seems to be the main obstacle to obtain a fully automated NNLO calculation framework similar to the one-loop case, that will satisfy the anticipated precision requirements at the LHC [20].

\section{The Simplified Differential Equations Approach}

In the last fifteen years, the calculation of virtual corrections has been revolutionized with the advent of automated reduction techniques to MI [15, 14, 16, 21] and the development of systematic solutions of differential equations [22, 23, 24, 25] satisfied by MI or the evaluation of their MellinBarnes representations [26, 27]. The differential equations approach (DE) has proven to be very powerful in a large number of computations, including two-loop four-point functions with massless and massive internal propagators. Within this framework, DE for the MI are derived, in terms of kinematical invariants. Assume that one is interested in calculating an $l$-loop Feynman integral 
with external momenta $\left\{p_{j}\right\}$ and internal propagators that are massless. Any $l$-loop Feynman integral can be then written as

$$
G_{a_{1} \cdots a_{n}}\left(\left\{p_{j}\right\}, \varepsilon\right)=\int\left(\prod_{r=1}^{l} \frac{d^{d} k_{r}}{i \pi^{d / 2}}\right) \frac{1}{D_{1}^{a_{1}} \cdots D_{n}^{a_{n}}}, \quad D_{i}=\left(c_{i j} k_{j}+d_{i j} p_{j}\right)^{2}, d=4-2 \varepsilon
$$

with matrices $\left\{c_{i j}\right\}$ and $\left\{d_{i j}\right\}$ determined by the topology and the momentum flow of the graph, and the denominators are defined in such a way that all scalar product invariants can be written as a linear combination of them. The exponents $a_{i}$ are integers and may be negative in order to accommodate irreducible numerators. Any integral $G_{a_{1} \cdots a_{n}}$ can be written as a linear combination of a finite subset of such integrals, called Master Integrals, with coefficients depending on the independent scalar products, $s_{i j}=p_{i} \cdot p_{j}$, and space-time dimension $d$, by the use of integration by parts identities $[15,14]$. In the traditional DE method, the MI $\vec{G}^{M I}\left(\left\{s_{i j}\right\}, \varepsilon\right)$, are differentiated with respect to $p_{i} \cdot \frac{\partial}{\partial p_{j}}$, and the resulting integrals are reduced by IBP to give a linear system of DE for $\vec{G}^{M I}\left(\left\{s_{i j}\right\}, \varepsilon\right)[22,28]$. The invariants, $s_{i j}$, are then parametrised in terms of dimensionless variables, defined on a case by case basis, so that the resulting DE can be solved in terms of Goncharov Polylogarithms (GPs) [29, 30].The GPs are defined recursively as follows

$$
\mathscr{G}\left(a_{n}, \ldots, a_{1}, x\right)=\int_{0}^{x} d t \frac{1}{t-a_{n}} \mathscr{G}\left(a_{n-1}, \ldots, a_{1}, t\right)
$$

with the special cases, $\mathscr{G}(x)=1$ and $\mathscr{G}(\overbrace{0, \ldots, 0}^{n}, x)=\frac{1}{n !} \log ^{n}(x)$. Usually boundary terms corresponding to the appropriate limits of the chosen parameters have to be calculated using for instance expansion by regions techniques $[31,32]$.

SDE approach [33] is an attempt not only to simplify, but also to systematize, as much as possible, the derivation of the appropriate system of DE satisfied by the MI. To this end the external incoming momenta are parametrized linearly in terms of $x$ as $p_{i}(x)=p_{i}+(1-x) q_{i}$, where the $q_{i}$ 's are a linear combination of the momenta $\left\{p_{i}\right\}$ such that $\sum_{i} q_{i}=0$. If $p_{i}^{2}=0$, the parameter $x$ captures the off-shell-ness of the external legs. The class of Feynman integrals in (2.1) are now dependent on $x$ through the external momenta:

$$
G_{a_{1} \cdots a_{n}}\left(\left\{s_{i j}\right\}, \varepsilon ; x\right)=\int\left(\prod_{r=1}^{l} \frac{d^{d} k_{r}}{i \pi^{d / 2}}\right) \frac{1}{D_{1}^{a_{1}} \cdots D_{n}^{a_{n}}}, \quad D_{i}=\left(c_{i j} k_{j}+d_{i j} p_{j}(x)\right)^{2} .
$$

By introducing the dimensionless parameter $x$, the vector of MI $\vec{G}^{M I}\left(\left\{s_{i j}\right\}, \varepsilon ; x\right)$, which now depends on $x$, satisfies

$$
\frac{\partial}{\partial x} \vec{G}^{M I}\left(\left\{s_{i j}\right\}, \varepsilon ; x\right)=\mathbf{H}\left(\left\{s_{i j}\right\}, \varepsilon ; x\right) \vec{G}^{M I}\left(\left\{s_{i j}\right\}, \varepsilon ; x\right)
$$

a system of differential equations in one independent variable. Experience up to now shows that this simple parametrization can be used universally to deal with up to six kinematical scales involved $[33,34,35]$. The expected benefit is that the integration of the DE naturally captures the expressibility of MI in terms of GPs and more importantly makes the problem independent on the number of kinematical scales (independent invariants) involved. Note that as $x \rightarrow 1$, the original configuration of the loop integrals (2.1) is reproduced, which eventually corresponds to a simpler one with one scale less. 


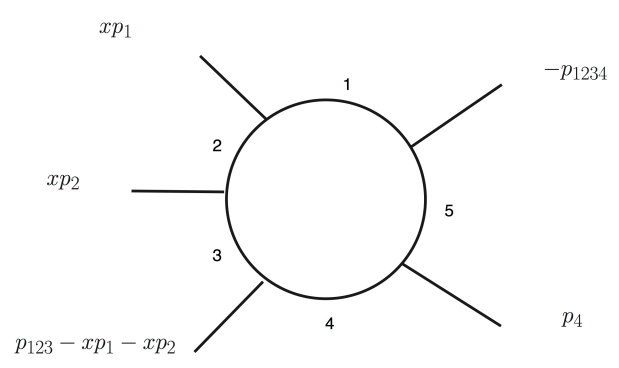

Figure 1: The one-loop pentagon graph with one off-shell leg, $G_{1111}$. The labels refer to the denominators in Eq.(3.1).

\section{Master Integrals calculations}

The SDE approach has been proven useful in calculations of MI at one and two loops.

\subsection{One-loop pentagon at $O(\varepsilon)$}

The one-loop pentagon presented in Fig.1 is given by

$$
G_{11111}=\int \frac{d^{d} k}{i \pi^{d / 2}} \frac{1}{\left(-k^{2}\right)\left(-\left(k+x p_{1}\right)^{2}\right)\left(-\left(k+x p_{12}\right)^{2}\right)\left(-\left(k+p_{123}\right)^{2}\right)\left(-\left(k+p_{1234}\right)^{2}\right)}
$$

with $p_{i}^{2}=0, i=1, \ldots, 4$, the obvious notation $p_{i \ldots j}=p_{i}+\ldots+p_{j}$ and $p_{1234}^{2}=\left(-p_{5}\right)^{2}=0$. The $\mathrm{DE}$ for the 5-point MI involves the following set of MI: four four-point $G_{11110}, G_{11101}, G_{11011}$, $G_{10111}$, one three-point, $G_{10110}$ and three two-point MI, $G_{00110}, G_{10010}, G_{10100}$. The two-point MI are known in a closed form in $\varepsilon$. All boundary conditions have been calculated by the DE, as described in [33]. The result for the one-mass pentagon up to order $\varepsilon$, is given by

$$
G_{11111}(x)=\frac{c_{\Gamma}}{x^{2} s_{23} s_{34} s_{45}}\left(1-\frac{1}{r_{1}}\right)\left(1-\frac{1}{r_{2}}\right)\left(1-\frac{x}{r_{1}}\right)^{-1-\varepsilon}\left(1-\frac{x}{r_{2}}\right)^{-1-\varepsilon} \sum_{i=-2}^{1} \varepsilon^{i} f_{i}
$$

where the quantities $r_{1}, r_{2}, c_{\Gamma}$ and $f_{i}$ can be found in [33]. Taking the limit $x \rightarrow 1$ from the previous expression, we get the result for the on-shell pentagon up to order $\varepsilon$, that is given by

$$
G_{1111}(1)=\frac{c_{\Gamma}}{s_{12} s_{23} s_{34} s_{45} s_{51}} \sum_{i=-2}^{1} \varepsilon^{i} f_{i}^{\prime}
$$

where again the $f_{i}^{\prime}$ can be found in [33].

\subsection{Full set of massless double-box MI with two off-shell legs}

There are in total six families of MI whose members with the maximum amount of denominators are graphically shown in Fig.2 and Fig.3. Three of these, Fig.2, contain only planar MI and will therefore be referred to as the planar families. They will be denoted by $P_{12}, P_{13}$ and $P_{23}$ [36] and contain 31,29 and $28 \mathrm{MI}$ respectively. The other three families, shown in Fig.3, contain planar MI with up to 6 denominators as well as non-planar MI with 6 and 7 denominators and will be referred to as the non-planar families of MI. These non-planar families will be denoted by $N_{12}, N_{13}$ 

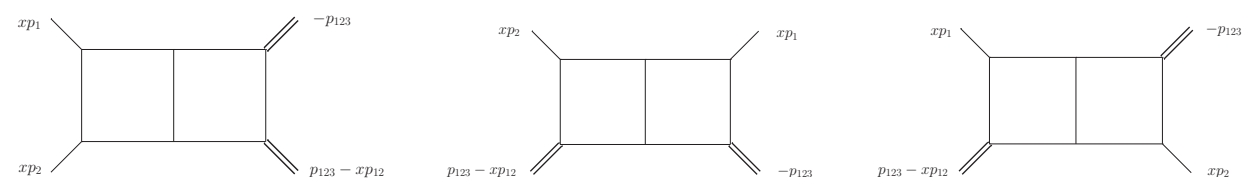

Figure 2: The parametrization of external momenta for the three planar double boxes of the families $P_{12}$ (left), $P_{13}$ (middle) and $P_{23}$ (right) contributing to pair production at the LHC. All external momenta are incoming.
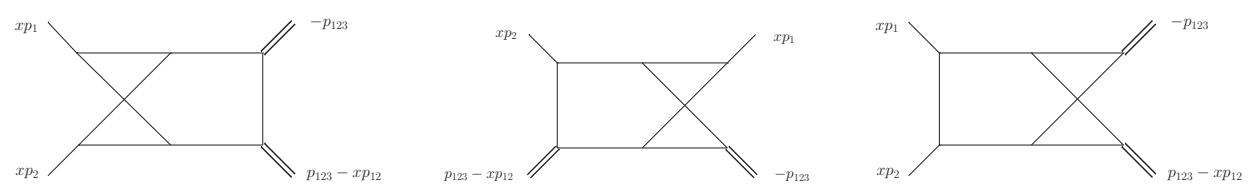

Figure 3: The parametrization of external momenta for the three non-planar double boxes of the families $N_{12}$ (left), $N_{13}$ (middle) and $N_{34}$ (right) contributing to pair production at the LHC. All external momenta are incoming.

and $N_{34}$ [37] and contain 35, 43 and 51 MI respectively. We have used both FIRE [38] and Reduze 2 [39] to perform the IBP reduction to the MI.

For instance the integrals in the family $P_{12}$ are given by

$$
\begin{aligned}
G_{a_{1} \cdots a_{9}}^{P_{12}}(x, s, \varepsilon):= & e^{2 \gamma_{E} \varepsilon} \int \frac{d^{d} k_{1}}{i \pi^{d / 2}} \frac{d^{d} k_{2}}{i \pi^{d / 2}} \frac{1}{k_{1}^{2 a_{1}}\left(k_{1}+x p_{1}\right)^{2 a_{2}}\left(k_{1}+x p_{12}\right)^{2 a_{3}}\left(k_{1}+p_{123}\right)^{2 a_{4}}} \\
& \times \frac{1}{k_{2}^{2 a_{5}}\left(k_{2}-x p_{1}\right)^{2 a_{6}}\left(k_{2}-x p_{12}\right)^{2 a_{7}}\left(k_{2}-p_{123}\right)^{2 a_{8}}\left(k_{1}+k_{2}\right)^{2 a_{9}}},
\end{aligned}
$$

where the letters appearing as weights in GPs are given by

$$
I\left(P_{12}\right)=\left\{0,1, \frac{q}{s_{12}}, \frac{s_{12}}{q}, \frac{q}{q-s_{23}}, 1-\frac{s_{23}}{q}, 1+\frac{s_{23}}{s_{12}}, \frac{s_{12}}{s_{12}+s_{23}}\right\}
$$

with $s_{12}:=p_{12}^{2}, s_{23}:=p_{23}^{2}, q:=p_{123}^{2}, p_{i}^{2}=0$. Details of the calculations as well as the results in terms of GPs for all families can be found in [34].

In order to compute the MI in arbitrary kinematics, especially in the physical region, the GPs have to be properly analytically continued. In general all variables, including the momenta invariants $s_{i j}\left(s_{12}, s_{23}\right.$ and $q$ in the present study) and the parameter $x$, would acquire an infinitesimal imaginary part, $s_{i j} \rightarrow s_{i j}+i \delta_{s_{i j}} \eta, x \rightarrow x+i \delta_{x} \eta$, with $\eta \rightarrow 0$. The parameters $\delta_{s_{i j}}$ and $\delta_{x}$ are determined as follows: the first class of constraints on the above-mentioned parameters originates form the input data to the DE, namely the one-scale master integrals that need to be properly defined in each kinematical region. The second class of constraints results from the second graph polynomial [40], $\mathscr{F}$, which after expressed in terms of $s_{i j}$ and $x$, should acquire a definite-negative imaginary part in the limit $\eta \rightarrow 0$. Combining these two classes of constraints on the parameters $\delta_{s_{i j}}$ and $\delta_{x}$, the imaginary part of all the GPs involved is fixed and we have checked that the result for the MI is identical in the limit $\eta \rightarrow 0$ and moreover agrees with the one obtained by other calculations. We have performed several numerical checks of all our calculations. The numerical results have been compared with those provided by the numerical code SecDec [41, 42, 43] in the 

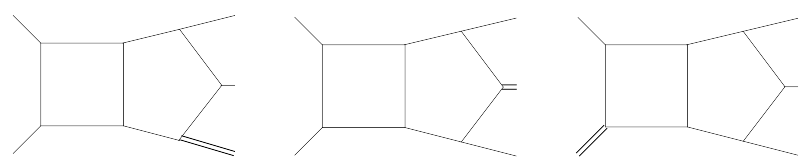

Figure 4: The three planar pentaboxes of the families $P_{1}$ (left), $P_{2}$ (middle) and $P_{3}$ (right) with one external massive leg.

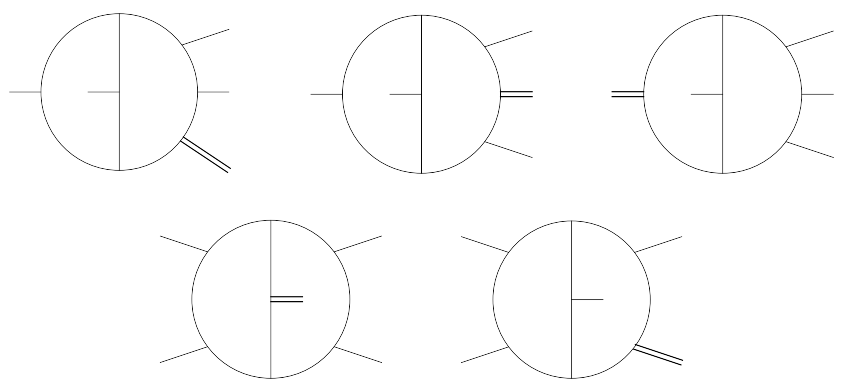

Figure 5: The five non-planar families with one external massive leg.

Euclidean region and with analytic results presented in $[36,37]$ for the physical region. In all cases we find perfect agreement and reference numerical results can be found in the ancillary files in [34].

\subsection{Massless pentabox MI with up to one off-shell leg}

For the massless pentabox MI with one off-shell leg, there are in total three families of planar MI whose members with the maximum amount of denominators, namely eight, are graphically shown in Fig.4. Similarly, there are five non-planar families of MI as given in Fig.5. We have checked that the other five-point integrals with one massive external leg are reducible to $\mathrm{MI}$ in one of these eight MI families. The two-loop planar and non-planar diagrams have not been calculated yet; in [35] we have recently completed the calculation of the $P_{1}$ family (Fig.4). In fact by taking the limit $x \rightarrow 1$ all planar graphs for massless on-shell external momenta have been derived as well ${ }^{1}$. We have used the c++ implementation of the program FIRE [45] to perform the IBP reduction to the set of MI in $P_{1}$.

For the family of integrals $P_{1}$ the external momenta are parametrized in $x$ as shown in Fig.6. The parametrization is chosen such that the double box MI with two massive external legs that is contained in the family $P_{1}$ has exactly the same parametrization as that one chosen in [34], i.e. two massless external momenta $x p_{1}$ and $x p_{2}$ and two massive external momenta $p_{123}-x p_{12}$ and $-p_{123}$. The MI in the family $P_{1}$ are therefore a function of a parameter $x$ and the following five invariants: $s_{12}:=p_{12}^{2}, s_{23}:=p_{23}^{2}, s_{34}:=p_{34}^{2}, s_{45}:=p_{45}^{2}=p_{123}^{2}, s_{51}:=p_{15}^{2}=p_{234}^{2}, p_{i}^{2}=0$, where the notation $p_{i \cdots j}=p_{i}+\cdots+p_{j}$ is used and $p_{5}:=-p_{1234}$. As the parameter $x \rightarrow 1$, the external momentum $q_{3}:=p_{123}-x p_{12}$ becomes massless, such that our parametrization also captures the on-shell case.

The resulting differential equation in matrix form can be written as

$$
\partial_{x} \mathbf{G}=\mathbf{M}\left(\left\{s_{i j}\right\}, \varepsilon, x\right) \mathbf{G}
$$

\footnotetext{
${ }^{1}$ Results related to massless planar pentabox appear also recently in [44].
} 


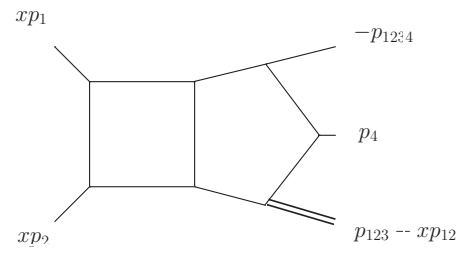

Figure 6: The parametrization of external momenta in terms of $x$ for the planar pentabox of the family $P_{1}$. All external momenta are incoming.

where $\mathbf{G}$ stands for the array of the $74 \mathrm{MI}$ involved in the family $P_{1}$. The twenty letters $l_{i}$ involved, are given in [35]. Although the DE can be solved starting from, e.g. (3.5), and the result can be expressed as a sum of GPs with argument $x$ and weights given by the letters $l_{i}$, it is more elegant and easy-to-solve to derive a Fuchsian system of equations [46], where only single poles in the variable $x$ will appear. A series of transformation described in [35], brings the system into the form

$$
\partial_{x} \mathbf{G}=\left(\varepsilon \sum_{i=1}^{19} \frac{\mathbf{M}_{i}}{\left(x-l_{i}\right)}\right) \mathbf{G}
$$

where the residue matrices $\mathbf{M}_{i}$ are independent of $x$ and $\varepsilon$. The result can be straightforwardly given as

$$
\begin{aligned}
\mathbf{G} & =\varepsilon^{-2} \mathbf{b}_{0}^{(-2)}+\varepsilon^{-1}\left(\sum \mathscr{G}_{a} \mathbf{M}_{a} \mathbf{b}_{0}^{(-2)}+\mathbf{b}_{0}^{(-1)}\right)+\left(\sum \mathscr{G}_{a b} \mathbf{M}_{a} \mathbf{M}_{b} \mathbf{b}_{0}^{(-2)}+\sum \mathscr{G}_{a} \mathbf{M}_{a} \mathbf{b}_{0}^{(-1)}+\mathbf{b}_{0}^{(0)}\right) \\
& +\varepsilon\left(\sum \mathscr{G}_{a b c} \mathbf{M}_{a} \mathbf{M}_{b} \mathbf{M}_{c} b_{0}^{(-2)}+\sum \mathscr{G}_{a b} \mathbf{M}_{a} \mathbf{M}_{b} \mathbf{b}_{0}^{(-1)}+\sum \mathscr{G}_{a} \mathbf{M}_{a} \mathbf{b}_{0}^{(0)}+\mathbf{b}_{0}^{(1)}\right) \\
& +\varepsilon^{2}\left(\sum \mathscr{G}_{a b c d} \mathbf{M}_{a} \mathbf{M}_{b} \mathbf{M}_{c} \mathbf{M}_{d} \mathbf{b}_{0}^{(-2)}+\sum \mathscr{G}_{a b c} \mathbf{M}_{a} \mathbf{M}_{b} \mathbf{M}_{c} \mathbf{b}_{0}^{(-1)}+\sum \mathscr{G}_{a b} \mathbf{M}_{a} \mathbf{M}_{b} \mathbf{b}_{0}^{(0)}+\sum \mathscr{G}_{a} \mathbf{M}_{a} \mathbf{b}_{0}^{(1)}+\mathbf{b}_{0}^{(2)}\right)
\end{aligned}
$$

with the arrays $\mathbf{b}_{0}^{(k)}, k=-2, \ldots, 2$ representing the $x$-independent boundary terms in the limit $x=0$ at order $\varepsilon^{k}$. The expression is in terms of Goncharov polylogarithms, $\mathscr{G}_{a b \ldots}=\mathscr{G}\left(l_{a}, l_{b}, \ldots ; x\right)$. Details on the calculation of boundary terms and of the $x \rightarrow 1$ limit can be found in [35] $]^{2}$.

The solution for all 74 MI contains $O(3,000)$ GPs which is roughly six times more than the corresponding double-box with two off-shell legs planar MI. We have performed several numerical checks of all our calculations. The numerical results, also included in the ancillary files [47], have been performed with the GiNaC library [48] and compared with those provided by the numerical code SecDec [41, 42, 43, 49] in the Euclidean region for all MI and in the physical region whenever possible (due to CPU time limitations in using SecDec) and found perfect agreement. For the physical region we are using the analytic continuation as described in the previous section as well as in [34]. At the present stage we are not setting a fully-fledged numerical implementation, which will be done when all families will be computed. Our experience with double-box computations show that using for instance Hyper Int [50] to bring all GPs in their range of convergence, before evaluating them numerically by $\mathbf{G i N a C}$, increases efficiency by two orders of magnitude. Moreover expressing GPs in terms of classical polylogarithms and $L i_{2,2}$, could also reduce substantially the CPU time [51]. Based on the above we estimate that a target of $O\left(10^{2}-10^{3}\right)$ milliseconds can be achieved.

\section{Outlook}

We have demonstrated that based on the Simplified Differential Equations approach [33] Master Integrals, including massless double-box with two off-shell legs and pentabox with up to one off-shell leg, can

\footnotetext{
${ }^{2}$ See also talk by C. Wever.
} 
be expressed in terms of Goncharov polylogarithms. The complexity of the resulting expressions is certainly promising that the project of computing all MI relevant to massless QCD, namely all eight-denominator MI with arbitrary configuration of the external momenta, is feasible. Having such a complete library of two-loop MI, the analog of $A_{0}, B_{0}, C_{0}, D_{0}$ scalar integrals at one loop, the reduction of an arbitrary two-loop amplitude à la OPP can pave the road for a NNLO automation in the near future.

As experience shows, there are several issues that will need to attract our attention in order to accomplish our goal. First of all in order to systematize the whole procedure of reducing an arbitrary Feynman Integral in terms of MI in an efficient way, a deepening of our current understanding of IBP identities [52, 53] is necessary [54]. Secondly, further standardising the procedure to obtain a canonical form of DE [55], which drastically simplifies the expression of MI in terms of GPs, is certainly a very desirable feature. Thirdly, the inclusion of MI with massive internal propagators, at a first stage with one mass scale corresponding to the heavy top quark, will provide the complete basis for NNLO QCD automated computations. Moreover, the calculation of boundary terms for the DE can benefit from further developments and exploitations of expansion-by-regions techniques, in conjunction with Mellin-Barnes representation of the resulting integrals. Finally, on the numerical side, a more efficient ${ }^{3}$ computation of polylogarithms is also necessary.

\section{References}

[1] J. R. Andersen et al., Les Houches 2013: Physics at TeV Colliders: Standard Model Working Group Report, arXiv:1405.1067.

[2] Z. Bern, L. J. Dixon, D. C. Dunbar, and D. A. Kosower, Fusing gauge theory tree amplitudes into loop amplitudes, Nucl.Phys. B435 (1995) 59-101, [hep-ph/9409265].

[3] Z. Bern, L. J. Dixon, D. C. Dunbar, and D. A. Kosower, One loop n point gauge theory amplitudes, unitarity and collinear limits, Nucl.Phys. B425 (1994) 217-260, [hep-ph / 9403226 ].

[4] G. Ossola, C. G. Papadopoulos, and R. Pittau, Reducing full one-loop amplitudes to scalar integrals at the integrand level, Nucl.Phys. B763 (2007) 147-169, [hep-ph/ 0609007 ].

[5] G. Ossola, C. G. Papadopoulos, and R. Pittau, On the Rational Terms of the one-loop amplitudes, JHEP 0805 (2008) 004, [arXiv: 0802 . 1876].

[6] SM AND NLO MULTILEG and SM MC Working Groups Collaboration, J. Alcaraz Maestre et al., The SM and NLO Multileg and SM MC Working Groups: Summary Report, arXiv:1203.6803.

[7] R. K. Ellis, Z. Kunszt, K. Melnikov, and G. Zanderighi, One-loop calculations in quantum field theory: from Feynman diagrams to unitarity cuts, Phys.Rept. 518 (2012) 141-250, [arXiv:1105.4319].

[8] J. Gluza, K. Kajda, and D. A. Kosower, Towards a Basis for Planar Two-Loop Integrals, Phys.Rev. D83 (2011) 045012, [arXiv: 1009 .0472].

[9] D. A. Kosower and K. J. Larsen, Maximal Unitarity at Two Loops, Phys.Rev. D85 (2012) 045017 , [arXiv:1108.1180].

[10] P. Mastrolia and G. Ossola, On the Integrand-Reduction Method for Two-Loop Scattering Amplitudes, JHEP 1111 (2011) 014, [arXiv:1107.6041].

[11] S. Badger, H. Frellesvig, and Y. Zhang, Hepta-Cuts of Two-Loop Scattering Amplitudes, JHEP 1204 (2012) 055, [arXiv:1202.2019].

[12] S. Badger, H. Frellesvig, and Y. Zhang, A Two-Loop Five-Gluon Helicity Amplitude in QCD, JHEP 1312 (2013) 045, [arXiv:1310.1051].

\footnotetext{
${ }^{3}$ See talk by D. Tommasini and recent work in [56].
} 
[13] C. Papadopoulos, R. Kleiss, and I. Malamos, Reduction at the integrand level beyond NLO, PoS Corfu2012 (2013) 019.

[14] F. Tkachov, A Theorem on Analytical Calculability of Four Loop Renormalization Group Functions, Phys.Lett. B100 (1981) 65-68.

[15] K. Chetyrkin and F. Tkachov, Integration by Parts: The Algorithm to Calculate beta Functions in 4 Loops, Nucl.Phys. B192 (1981) 159-204.

[16] V. A. Smirnov, Evaluating Feynman integrals, Springer Tracts Mod. Phys. 211 (2004) 1-244.

[17] C. Anastasiou and A. Lazopoulos, Automatic integral reduction for higher order perturbative calculations, JHEP 07 (2004) 046, [hep-ph / 0404258 ].

[18] A. V. Smirnov and M. N. Tentyukov, Feynman Integral Evaluation by a Sector decomposiTion Approach (FIESTA), Comput. Phys. Commun. 180 (2009) 735-746, [arXiv : 0807.4129 ].

[19] G. 't Hooft and M. Veltman, Scalar One Loop Integrals, Nucl.Phys. B153 (1979) 365-401.

[20] J. Butterworth, G. Dissertori, S. Dittmaier, D. de Florian, N. Glover, et al., Les Houches 2013: Physics at TeV Colliders: Standard Model Working Group Report, arXiv:1405.1067.

[21] S. Laporta, High precision calculation of multiloop Feynman integrals by difference equations, Int. J. Mod. Phys. A15 (2000) 5087-5159, [hep-ph/ 0102033$].$

[22] A. Kotikov, Differential equations method: New technique for massive Feynman diagrams calculation, Phys.Lett. B254 (1991) 158-164.

[23] Z. Bern, L. J. Dixon, and D. A. Kosower, Dimensionally regulated one loop integrals, Phys.Lett. B302 (1993) 299-308, [hep-ph/9212308].

[24] Z. Bern, L. J. Dixon, and D. A. Kosower, Dimensionally regulated pentagon integrals, Nucl. Phys. B412 (1994) 751-816, [hep-ph/9306240].

[25] T. Gehrmann and E. Remiddi, Differential equations for two loop four point functions, Nucl.Phys. B580 (2000) 485-518, [hep-ph/9912329].

[26] V. A. Smirnov, Analytical result for dimensionally regularized massless on shell double box, Phys. Lett. B460 (1999) 397-404, [hep-ph/ 9905323$].$

[27] J. B. Tausk, Nonplanar massless two loop Feynman diagrams with four on-shell legs, Phys. Lett. B469 (1999) 225-234, [hep-ph/9909506].

[28] E. Remiddi, Differential equations for Feynman graph amplitudes, Nuovo Cim. A110 (1997) 1435-1452, [hep-th/9711188].

[29] A. B. Goncharov, Multiple polylogarithms, cyclotomy and modular complexes, Math.Res.Lett. 5 (1998) 497-516, [arXiv:1105.2076].

[30] A. B. Goncharov, Multiple polylogarithms, cyclotomy and modular complexes, math/ 0103059.

[31] M. Beneke and V. A. Smirnov, Asymptotic expansion of Feynman integrals near threshold, Nucl.Phys. B522 (1998) 321-344, [hep-ph/9711391].

[32] V. A. Smirnov, Applied asymptotic expansions in momenta and masses, Springer Tracts Mod.Phys. 177 (2002) 1-262.

[33] C. G. Papadopoulos, Simplified differential equations approach for Master Integrals, JHEP 1407 (2014) 088, [arXiv:1401.6057].

[34] C. G. Papadopoulos, D. Tommasini, and C. Wever, Two-loop Master Integrals with the Simplified Differential Equations approach, JHEP 01 (2015) 072, [arXiv: 1409.6114 ].

[35] C. G. Papadopoulos, D. Tommasini, and C. Wever, The Pentabox Master Integrals with the Simplified Differential Equations approach, arXiv:1511.0940. 
[36] J. M. Henn, K. Melnikov, and V. A. Smirnov, Two-loop planar master integrals for the production of off-shell vector bosons in hadron collisions, JHEP 1405 (2014) 090, [arXiv : 1402 . 7078 ].

[37] F. Caola, J. M. Henn, K. Melnikov, and V. A. Smirnov, Non-planar master integrals for the production of two off-shell vector bosons in collisions of massless partons, arXiv: 1404.5590.

[38] A. Smirnov, Algorithm FIRE - Feynman Integral REduction, JHEP 0810 (2008) 107, [arXiv:0807.3243].

[39] A. von Manteuffel and C. Studerus, Reduze 2 - Distributed Feynman Integral Reduction, arXiv: 1201.4330.

[40] C. Bogner and S. Weinzierl, Feynman graph polynomials, Int.J.Mod.Phys. A25 (2010) 2585-2618, [arXiv:1002.3458].

[41] T. Binoth and G. Heinrich, An automatized algorithm to compute infrared divergent multiloop integrals, Nucl.Phys. B585 (2000) 741-759, [hep-ph/ 0004013 ].

[42] G. Heinrich, Sector Decomposition, Int.J.Mod.Phys. A23 (2008) 1457-1486, [a rXiv: 0803 . 4177].

[43] S. Borowka, J. Carter, and G. Heinrich, Numerical Evaluation of Multi-Loop Integrals for Arbitrary Kinematics with SecDec 2.0, Comput.Phys.Commun. 184 (2013) 396-408, [arXiv: 1204.4152 ].

[44] T. Gehrmann, J. M. Henn, and N. A. L. Presti, Analytic form of the two-loop planar five-gluon all-plus-helicity amplitude in QCD, arXiv:1511.0540.

[45] A. V. Smirnov, FIRE5: a C++ implementation of Feynman Integral REduction, Comput. Phys. Commun. 189 (2014) 182-191, [arXiv: 1408 .2372].

[46] J. M. Henn, Lectures on differential equations for Feynman integrals, J. Phys. A48 (2015) 153001, [arXiv:1412.2296].

[47] available at https://www.dropbox.com/s/90iiqfcazrhwtso/results.tgz?dl=0.

[48] J. Vollinga and S. Weinzierl, Numerical evaluation of multiple polylogarithms, Comput.Phys.Commun. 167 (2005) 177, [hep-ph/ 0410259 ].

[49] S. Borowka, G. Heinrich, S. P. Jones, M. Kerner, J. Schlenk, and T. Zirke, SecDec-3.0: numerical evaluation of multi-scale integrals beyond one loop, Comput. Phys. Commun. 196 (2015) 470-491, [arXiv:1502.0659].

[50] E. Panzer, Algorithms for the symbolic integration of hyperlogarithms with applications to Feynman integrals, Comput. Phys. Commun. 188 (2014) 148-166, [arXiv: 1403.3385$].$

[51] T. Gehrmann, A. von Manteuffel, and L. Tancredi, The two-loop helicity amplitudes for $q \bar{q}^{\prime} \rightarrow V_{1} V_{2} \rightarrow 4$ leptons, JHEP 09 (2015) 128, [arXiv: 1503.0481 ].

[52] P. A. Baikov, Explicit solutions of the multiloop integral recurrence relations and its application, Nucl. Instrum. Meth. A389 (1997) 347-349, [hep-ph/9611449].

[53] V. A. Smirnov and M. Steinhauser, Solving recurrence relations for multiloop Feynman integrals, Nucl. Phys. B672 (2003) 199-221, [hep-ph/ 0307088$].$

[54] H. Ita, Two-loop Integrand Decomposition into Master Integrals and Surface Terms, arXiv:1510.0562.

[55] J. M. Henn, Multiloop integrals in dimensional regularization made simple, Phys.Rev.Lett. 110 (2013), no. 25 251601, [arXiv:1304.1806].

[56] H. Frellesvig, D. Tommasini, and C. Wever, On the reduction of generalized polylogarithms to $\mathrm{Li}_{n}$ and $\mathrm{Li}_{2,2}$ and on the evaluation thereof, arXiv:1601.0264. 